\title{
Lentos, estéticos y memoriosos. Las automovilidades lowriders y las estéticas de la nostalgia en Tijuana
}

\author{
Juan Antonio del Monte Madrigal
}

Los lowriders son grupos urbanos que se visibilizan al circular por las calles de la ciudad en un automóvil antiguo modificado con un complejo sistema hidráulico que permite bajar el auto a nivel del piso o subirlo a más de medio metro. Es un fenómeno transfronterizo cuyas prácticas están asociadas a la identidad chicana, incorpora elementos simbólicos de los pachucos y los cholos, y utiliza el automóvil como el mecanismo central de expresión de la simbología compartida. El presente trabajo analiza la construcción de identidades lowrider en Tijuana, Baja California, y el reforzamiento de las mismas a través de su exhibición performática en el espacio urbano. Se presentan los resultados de un trabajo de investigación en relación con las implicaciones de "automovilizar" las identidades.

PALABRAS CLAVE: identidades, automovilidades, lowriders, performance, nostalgia

\section{Slow, Aesthetic and Memorious. Lowrider Automobilities and the Aestethics of Nos- talgia in Tijuana}

Lowriders are urban groups that become visible circulating the city streets in old cars modified with a complex hydraulic system that allow them to lower the car to floor level or lift it to a couple of feet. This is a border phenomenon whose practices are related to chicano identity, include pachuco and cholo symbolic elements, and use the car as the central mechanism of expression of the shared symbols. This paper analyzes the construction of lowrider identities in Tijuana, Baja California, and the strengthening of them through their performative exhibition in the urban space. This text presents the results of a research project regarding the implications of "automobilizing" identities.

KEYWORDS: identities, automobilities, lowriders, performance, nostalgia 


\section{INTRODUCCIÓN}

$\mathrm{L}$ a heterogeneidad es uno de los rasgos distintivos de las ciudades modernas. Los espacios citadinos conjugan, interrelacionan y sobreponen distintas prácticas sociales que hacen evidente la pluralidad de mundos culturales que interactúan en las sociedades urbanas. En este sentido, la ciudad puede ser pensada como corolario de la convivencia de diferentes expresiones y performatividades culturales. Entender la ciudad desde una noción dinámica, relacional y práctica, es decir, embebida en formas plurales de vivirla y construirla, coloca a la circulación como una característica central de la misma que no puede ser obviada por los estudios antropológicos y nos invita a reparar en la urbe como un espacio de movilidades semantizadas por los grupos sociales que la habitan.

Los vehículos motorizados se han establecido a lo largo del siglo xx y principios del xxI como los protagonistas de la circulación de la vida urbana. Es prácticamente imposible pensar el desplazamiento en las ciudades modernas sin el uso de automóviles. Pero éstos no son sólo máquinas con fines utilitarios para desplazarse en la ciudad. Varios estudios han mostrado cómo las tecnologías de la automovilidad también fungen como vehículos de expresión de símbolos y significados culturales (Miller, 2001; Wollen y Kerr, 2002; García, Castellanos y Mantecón, 1996; Featherstone, Thrift y Urry, 2005). Las calles de Tijuana reflejan una marcada diversidad, robustecida sobre todo por su condición fronteriza. Dentro de esta pluralidad de formas de reconocimiento sociocultural, los lowriders son un conspicuo fenómeno identitario que forma parte de las dinámicas más visibles y expresivas que circulan en la ciudad. Los lowriders son un colectivo social que se distingue por hacer del automóvil antiguo modificado y su circulación en las calles de la urbe el elemento de mediación más importante para su adscripción identitaria.
En este texto se planteará la articulación de los factores involucrados en el establecimiento, el desarrollo y la circulación de las identidades adscritas a los mundos lowrider por la ciudad fronteriza de Tijuana. Para tratar de entender la construcción de pertenencias sociales asociadas a los vehículos motorizados a través del engranaje conceptual de las identidades y la automovilidad se presentarán los resultados de un estudio de caso llevado a cabo en la ciudad de Tijuana en torno a las tres movilidades de los lowriders, los significados de nostalgia que les atribuyen y las implicaciones de activar, por medio de estas prácticas, la memoria social.

\section{LA TIJUANA DE LOS CARROS}

Tijuana es una ciudad creada desde sus orígenes en el diseño del automóvil. En gran medida la vida cotidiana de la urbe se sustenta en el uso del carro. Los automóviles están insertos en el centro de la sociabilidad y la comunicación, lo que posibilita redes y vínculos comunicativos de interacción e impele a la vida social urbana hacia las formas de movilidad que se llevan a cabo desde los vehículos motorizados. Según el último censo del Instituto Nacional de Estadística y Geografía (INEGI), en 2010 Tijuana tenía un total de 704127 vehículos motorizados empadronados en el registro vehicular frente a poco más de un millón y medio de habitantes contabilizados, en tanto que en metrópolis con más de cuatro millones de habitantes, como Guadalajara y Monterrey, el padrón registra 834032 y 621383 vehículos, respectivamente. Es decir que mientras en estas ciudades hay un carro por cada cinco o seis habitantes, en Tijuana hay un carro por cada dos personas. Este dato comparativo da indicios de la centralidad de los vehículos motorizados en la vida de su población y del acceso que se tiene a ellos en la ciudad.

La proliferación de automóviles en Tijuana encuentra un punto de expansión a partir de diversos 
factores asociados a su situación fronteriza, entre los cuales destacan la planificación urbana y la experiencia del régimen fiscal de zona libre. Por un lado, la planificación y el diseño urbano de Tijuana fueron pensados para que el carro fuera, como en efecto es, un protagonista en sus dinámicas cotidianas, lo que emula en gran parte al diseño de la ciudad vecina, San Diego (Padilla, 1998). El crecimiento que tuvo la ciudad a partir de mediados del siglo anterior impulsó la construcción de desarrollos urbanos comerciales, como la Zona Río, y habitacionales, como la Zona Este o el actual desarrollo del Valle de las Palmas, donde se colocó al automóvil como la figura protagónica en el diseño y planificación del trazado. Avenidas anchas, enormes espacios de estacionamiento y una gran cantidad de vehículos motorizados pueblan el paisaje urbano de Tijuana. De hecho, el gran proyecto de urbanización de las dos últimas administraciones ha sido una repavimentación integral de las avenidas de la ciudad. En 2010 el presidente municipal declaró que "el Pire [Programa Integral de Repavimentación] representa el 43 o 44\% de todo el volumen de obra [pública municipal] que se hizo en Tijuana en tres años" (Ramos, 2010: 68), y anunció con ello el incentivo para el uso de vehículos motorizados en las calles de Tijuana. ${ }^{1}$

Por otro lado, el régimen de zona libre al que están adscritos los territorios fronterizos alude a una región con tratamientos fiscales especiales. Uno de sus beneficios es la exención parcial o total de los impuestos de importación. Esta disposición implica libertad arancelaria para el flujo de ciertos productos en la frontera, que incluye un régimen

\footnotetext{
${ }^{1}$ Con esto no se afirma que se "puede" circular en automóvil en toda la ciudad. Lo que se enfatiza es la idea, que atraviesa la implementación de políticas públicas, como lo mienta el testimonio anterior, de que la punta de desarrollo de la ciudad en materia estructural debe ser la plena circulación automovilística. Un acercamiento a las colonias marginadas de Tijuana, cuyos pobladores viven en una situación de extrema precariedad, bastará para darnos cuenta de que dicha planificación urbana genera procesos de exclusión social y de territorialización marcados por la condición de clase.
}

especial de importación de vehículos extranjeros. Tijuana es una ciudad volcada hacia la circulación automovilística y sus condiciones estructurales han sido diseñadas para fomentar la movilidad urbana de los vehículos motorizados. El contexto infraestructural que incita la circulación motorizada por la ciudad e incide en la misma se articula con su situación fronteriza, lo que da por resultado un escenario fértil para la conformación de grupos sociales que toman como referente de identidad a un automóvil que circula por la ciudad. Los lowriders surgen en estos escenarios fronterizos en condiciones que es preciso atender desde un análisis sociocultural, pues se visibilizan como manifestaciones populares que acarrean estigmas anclados en condiciones étnicas y de clase al compartir la simbología de cholos y pachucos. En este artículo enfatizamos el impulso exhibicionista de estos grupos cuando performan sus identidades a través de mecanismos de movilidad y exposición de sus características definitorias, los cuales funcionan como estrategias de redención de los estigmas, de resistencia social y de búsqueda de un lugar en una sociedad que históricamente ha invisibilizado sus prácticas.

\section{EL DEVENIR DEL LOWRIDER COMO FENÓMENO TRANSFRONTERIZO}

Los lowriders conforman un colectivo social cuyas prácticas de modificación del automóvil antiguo y sus formas de circulación en las calles - lo que aquí llamamos "automovilidades" - se han integrado como referentes importantes de sus características identitarias. Los mundos lowrider forman parte de un largo proceso que se entreteje con la historia del pueblo chicano en Estados Unidos, con la emergencia en el espacio público de manifestaciones culturales juveniles de mediados de siglo $\mathrm{xx}$ percibidas como una desviación social -en especial pachucos y cholos, con quienes comparten una simbología en común-, con la cultura automovilística del sur de California y con las relaciones 
sociales y comunicativas transfronterizas. La expresividad y simbología que el lowrider porta con sus carros cuando circula por la ciudad está asociada indefectiblemente con este entramado transfronterizo semantizado entre chicanos, pachucos, cholos y una cultura volcada hacia la automovilidad.

Los lowriders surgen como un elemento representativo de la identidad de la población del suroeste estadounidense chicano (Gradante, 1982; Plascencia, 1983; Bright, 1994; Tatum, 2011), comunidad cuya historia está plagada de prejuicios raciales y de clase, inmersa en la exclusión social, la cual han enfrentado con diversas estrategias de resistencia, desde posicionamientos políticos hasta prácticas culturales como las manifestaciones del pachuco, el cholo o el lowrider. La modificación de los carros antiguos asociada al movimiento Hot Rod de California, a la iconografía popularmexicana apropiada por los mexicoamericanos en Estados Unidos y a la expresividad y simbología de pachucos y cholos son elementos que configuran la representación y el performance del lowrider. El lowrider modifica y personaliza sus carros en una estética barroca y distintivamente chicana que se moviliza por la ciudad de manera muy lenta (Bright et al., 2000; Chavoya, 2004): modelos viejos, transformados para circular "bajito" o pegados al piso, interiores y exteriores pintados de manera elaborada que exaltan lo mexicano y lo popular y, como su característica principal, la instalación de bombas hidráulicas en los amortiguadores para que el carro pueda "bailar" y saltar de un lado a otro y de arriba para abajo. De esta manera, las prácticas de modificación del carro lowrider se insertaron como un elemento expresivo característico de las identidades mexicoamericanas y se posicionaron como formas para hacerse visibles públicamente.

La idea de tener el carro a sólo unas pulgadas del piso finca sus antecedentes en el pachuco car. Los pachucos fueron expuestos alrededor de la década de 1940 a una reprobación moral de la opinión pública por trastocar con sus prácticas, expresiones y performances los valores hegemónicos estadounidenses.
Sus principales características fueron las referencias biculturales, expresadas sobre todo en giros lingüísticos del español al inglés, los tatuajes y el traje zoot suit, lo que representa claramente una postura ante el statu quo de la moda estadounidense. Los pachucos incorporaron a su estilo y expresividad un repertorio simbólico vinculado a una forma de resistencia que incluía la modificación del pachuco car. Los pachucos bajaban sus carros con costales o ladrillos como una forma de distinguirse en las calles del este de Los Ángeles. Dicha práctica fue legislada como ilegal en California, por lo que desarrollaron un sistema de ascenso y descenso del automóvil a partir de la colocación de bombas hidráulicas en los amortiguadores (Sandoval, 2003), lo que marca el inicio del estilo lowrider. Por su parte:

los cholos aparecen en el ámbito cotidiano transfronterizo retomando códigos utilizados como recurso de resistencia social por el pachuco y una simbología popular-mexicana, reapropiada y recreada en el movimiento de resistencia político-cultural durante el movimiento chicano que se decantó en los barrios chicano-mexicanos en Estados Unidos (Valenzuela, Nateras y Reguillo, 2007: 42).

Jóvenes socializados en colonias populares surgidas en barrios chicanos de Los Ángeles, estigmatizados como desadaptados sociales cuyo peculiar vestuario, complejo lenguaje, gusto por la música antigua, tatuajes, murales y grafitis son a la vez símbolo de identificación ante "la raza" y de exclusión ante la sociedad (Valenzuela, 1988). El cholismo y el lowrider son movimientos asociados. Si bien unos se ubican en redes barriales y otros circulan en automóvil por la ciudad, tienen una simbología compartida que se reproduce en actitudes, vestuarios y murales que los últimos movilizan en sus carros.

A diferencia del Hot Rod californiano que enfatiza la funcionalidad del carro, ${ }^{2}$ el lowrider toma el

${ }^{2} \mathrm{El} \mathrm{Hot} \mathrm{Rod,} \mathrm{automóvil} \mathrm{creado} \mathrm{por} \mathrm{jóvenes} \mathrm{californianos} \mathrm{que} \mathrm{no}$ podían costearse uno nuevo, construido a partir de viejas carrocerías y partes de automóviles de depósitos de chatarra, fueron 


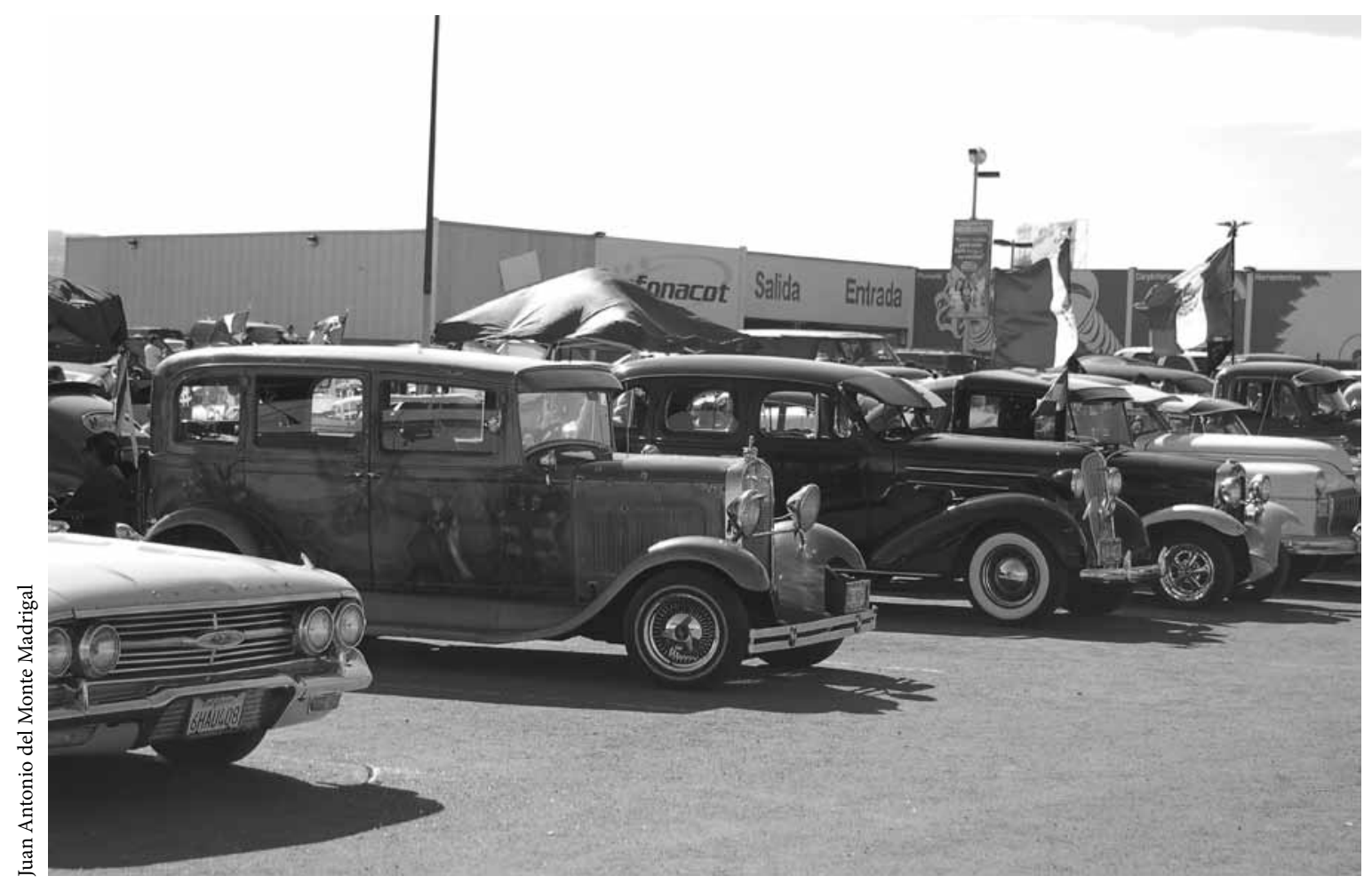

Lowriders, 2012.

camino opuesto y acentúa la exhibición y la contemplación, el parque y la calle del vecindario se convierten paulatinamente en sus lugares favoritos de "paseo". En lugar de motores alterados, poder y velocidad, el lowrider exhibe la lentitud, la estética y el ritmo. Esta diferencia ha sido interpretada también como una toma de distancia ante los valores dominantes de la cultura automovilística estadounidense, como la velocidad y el poderoso alzado de sus motores, por la parsimonia y lo "tumbado" (Bright, 1994; Sandoval, 2003; Tatum, 2011). La actitud "tumbado" es fundamental para el cholo

carros con motores alterados cuya característica principal fue la velocidad, el ruido, la decoración y el gran poder de sus motores. Esta práctica de creación y alteración automovilística es un antecedente de la modificación de carros que se lleva a cabo desde las culturas lowrider (Ganahl, 2000). Por ello, la utilización de un coche como el elemento central de la expresividad lowrider es deudora de la cultura californiana del automóvil. y alude a una condición de clase y estatus social precarizado (Valenzuela, 1988). De este modo, el distanciamiento que se plantea frente a la cultura dominante estadounidense a través de la exhibición de la "extravagancia" del carro es además una manera de buscar ingresar a espacios sociales en aquella sociedad. El lowrider plantea la exhibición de una tradición de resistencia cultural chicana que busca espacios de expresión dentro de las formas institucionales estadounidenses.

Con el antecedente de la tradición californiana de modificación de automóviles - como el Hot Rod-, la práctica del lowrider tiene su origen en asociación a la identidad chicana e incorpora elementos simbólicos del pachuco y el cholo -incluidos la música y el vestuario-, pero con el automóvil como el mecanismo central de expresión de la simbología compartida, que por otra parte tiene la importante cualidad de movilizarse públicamente 
por las calles de la ciudad. ${ }^{3}$ Tal como los pachucos y los cholos, los lowriders aparecen en escenarios transfronterizos donde se comparten códigos de manera recíproca en complejos procesos de interacción en los límites de dos naciones. En Tijuana las prácticas del lowrider decantaron en formas performáticas asociadas al movimiento de los carros por la ciudad y relacionadas con la simbología de las identidades juveniles del pachuco y el cholo. El proceso de recreación y resignificación de las prácticas del lowrider chicano en esta ciudad no es un mero reflejo de lo que ocurre del otro lado de la línea fronteriza, pues la vecindad entre México y Estados Unidos exhibe asimetrías y distinciones que implican procesos diferenciados de influencia e interacción sociocultural, es decir, dinámicas de resistencia, dominación, exclusión y apropiación. Los resultados del trabajo de campo que a continuación se presentan intentan mostrar cómo fue reapropiado el performance de los lowriders en su movimiento por la ciudad de Tijuana.

\section{IDENTIDADES Y AUTOMOVILIDAD EN LOS LOWRIDERS}

Los lowriders son un fenómeno identitario conformado por individuos que interiorizan el repertorio simbólico asociado a los mundos chicano, pachuco y cholo, dotan de sentido a las prácticas de modificación de carros y encuentran en su movilidad por la ciudad $-\mathrm{y}$ la consecuente visibilidad pública- el reconocimiento de ser expresiones que configuran el paisaje cultural y la coreografía urbana

\footnotetext{
${ }^{3}$ Se puede enunciar que las características que delinean la imagen y expresión de los colectivos lowrider aluden a la utilización de un automóvil antiguo modificado decorado con una estética mexicoamericana, al uso de vestimenta al estilo pachuco y a ciertas prácticas de estas culturas juveniles, como los "bailes" o el "paseo lento" por la ciudad - aspecto que se delineará más adelante con el análisis de sus automovilidades-. Estas características son apropiadas por los miembros de estas agrupaciones y se autoidentifican con ellas.
}

de Tijuana. La postura constructivista de las identidades entiende que éstas son históricas, relacionales y procesuales (Taylor, 1996; Bauman, 2001; Giménez, 2009). Las identidades son un proceso abierto y dinámico, no son una "esencia" ni un producto estático o acabado y se generan a partir de la interacción social semantizada. Las identidades, por tanto, hacen referencia a un proceso histórico de continuidades y discontinuidades en el cual el actor social se adscribe, se reconoce y se distingue de otros. De modo que las identidades son un proceso subjetivo de adscripción sociocultural, pero —en términos de Taylor- para que ésta sea "lograda" tiene que generarse una operación central en el proceso de construcción identitaria: el reconocimiento de los demás sujetos con quienes se establecen relaciones sociales y se interactúa. De aquí se desprende que las identidades son una dialéctica constante entre los procesos de definición interna y de imputación externa: "es una especie de compromiso o negociación entre autoafirmación y asignación identitaria, entre 'autoidentidad' y 'exoidentidad”' (Giménez, 2004: 90). Por un lado, los individuos se diferencian de otros sobre la base de un criterio de comunidad y de adscripción a un grupo; por otro, este proceso debe ser reconocido por los "otros" como una identidad. Este proceso de reconocimiento por los significados de adscripción identitaria muestra que las identidades no son planas, sino que están envueltas en una constante tensión y disputa que se lleva a cabo desde "posiciones relacionales de poder” (Valenzuela, 2000: 28). Así, la negociación por el reconocimiento - sea el que se pide o el que se otorga- se hace siempre desde una posición de poder.

La construcción del complejo simbólico-expresivo que distingue a los lowriders en la ciudad - principalmente compuesto por carros antiguos modificados- está basada en la posibilidad de ser movilizado y exhibido por la urbe, lo que constituye, en este sentido, la búsqueda del reconocimiento de su identidad por las calles de la ciudad. Las identidades lowrider se hacen reconocer en el espacio público a 
partir de una serie de estrategias de manifestación ligadas a la movilidad y a los actos performáticos de quienes manejan los vehículos motorizados. Tim Cresswell, en su libro On the Move (2006), menciona que el análisis de las movilidades no se restringe a sólo hablar de un desplazamiento sobre un tiempo y un espacio, sino que el tratamiento sociológico del fenómeno implica ubicar dichos desplazamientos sobre un tiempo social y un espacio social. Es decir, las movilidades están insertas en complejos procesos espacio-temporales de interacción social y el poder explicativo del análisis de dicha movilidad radica en abundar en torno a su significado. Las movilidades se llevan a cabo en un mundo lleno de significados sociales, de modo que los movimientos involucrados en éstas son simbolizados con sentidos socialmente construidos. En el caso del presente trabajo, tal construcción significativa de las movilidades lowrider corresponde al espacio y al tiempo de los escenarios fronterizos actuales.

John Urry (2007), por su parte, aporta la idea de que las relaciones sociales, así como la identidad, tienen lugar a través de redes de personas, objetos e ideas que, más que estar en un espacio delimitado, están movilizándose. Hablar de las movilidades implica ir más allá de la idea de que moverse es llegar de un lugar a otro para analizar el propio proceso de estar en movimiento y entender que dicho proceso implica estar envuelto en relaciones sociales e involucrado con objetos y/o, sobre todo, significados. Desde las teorías de las movilidades se propone enfocar el análisis en la articulación de personas con diferentes objetos para hablar de la posibilidad de convertirnos en seres móviles en el mundo contemporáneo, lo que ha decantado en conceptualizaciones como la de las automovilidades. Este término hace referencia a un sistema de movilización que se lleva a cabo desde vehículos motores que no sólo proveen movilidad y velocidad, sino que también representan un espacio personalizado y cómodo para el conductor y su acompañante - en caso de que exista-, que permite movilizarse de la manera y en la dirección que sea más placentera y significativa (Featherstone, Thrift y Urry, 2005). Mimi Sheller y John Urry definen la automovilidad en un artículo seminal para el tema como "una compleja amalgama que interconecta máquinas, prácticas sociales y modos de habitar, no en un espacio estacionario, sino en una cápsula móvil, semiprivada y altamente peligrosa" (Sheller y Urry, 2000: 739). ${ }^{4}$ El concepto de automovilidad funciona, prosiguen los autores, para hacer referencia a la capacidad de movilidad en conjunto con un objeto. Con ello se sugieren "complejas hibridaciones" (Urry, 2007: 50) en tanto se mueven sujetos y objetos en complejos sistemas de movilidad — como la ciudad y sus calles - a través de "marcos mundanos de movimiento" (Thrift, 2004: 583), como los vehículos motorizados modificados al estilo lowrider.

La circulación, entonces, se coloca como una característica central de la ciudad y ésta como un campo de movilidades significantes, "un torbellino de fuerzas e intensidades que atraviesan y relacionan todo tipo de actores, humanos y no humanos, en todas las combinaciones de su agencia" (Amin y Thrift, 2002: 83). Michel de Certeau (1996) decía que los transeúntes van construyendo con sus pasos el espacio urbano, y centra la discusión en el hecho de que la construcción del espacio se hace por medio de prácticas que lo cargan de significados, como caminar. Sin embargo, estas prácticas en la ciudad no se agotan en las de los transeúntes: "si la ciudad es un lenguaje hablado por caminantes, entonces la ciudad pospeatonal no sólo puede quedar en silencio, sino que está en riesgo de convertirse en un lenguaje muerto" (Thrift, 2005: 44). Las prácticas de manejar también tienen algo que decir en el espacio urbano, como lo hace ver el performance de los lowriders cuando conducen por la ciudad.

La importancia y pertinencia de analizar la circulación por las calles radica en que es justo en esas

\footnotetext{
${ }^{4}$ Todas las citas extraídas de textos que se presentan en inglés en la bibliografía fueron traducidas por el autor del presente artículo.
} 
ocasiones cuando los lowriders, como colectivos identitarios, se refuerzan al hacer público - de exhibir, mostrar y circular - todo el complejo simbólico-expresivo que han construido para formar un sentido de pertenencia. En estas circulaciones se reafirma la identidad motorizada de lowriders a partir del despliegue, la exhibición y la expresividad de su simbología. Así, performan de manera pública ese sentido de pertenencia a la colectividad lowrider. Recordemos que, como anota Jeffrey Alexander, el performance "es el proceso social por el que los actores, individual o colectivamente, despliegan para otros el significado de su situación social" (Alexander, 2011: 27). En esta movilidad pública de su expresividad se comunica y refuerza su identidad.

\section{LA TERNA AUTOMÓVIL DE LOS LOWRIDERS: LENTOS, ESTÉTICOS Y MEMORIOSOS}

Las identidades lowrider en la ciudad de Tijuana muestran una continuidad con la simbología del pachuco y el cholo, expresiones asociadas en cuanto a iconografía, pero diferentes en cuanto a prácticas. ${ }^{5}$ Por ello, nos proponemos analizar dichas

\footnotetext{
${ }^{5}$ Es precisamente su relación simbólica con el pachuco y el cholo y el hecho de haber surgido en barrios populares ligados a estas culturas juveniles - como la colonia Libertad en Tijuana- lo que le ha acarreado el mayor peso estigmatizante a su expresividad. Según los testimonios del trabajo de campo, la asociación que se hacía al referir a los lowriders de la colonia Libertad como pachucos era representarlos de manera peyorativa como drogadictos: "mucha gente nomás podía decir que éramos unos mariguanos pachucos, que era la palabra de la alta sociedad" (Jinete, entrevista) (Del Monte, 2012). Sin embargo, actualmente los lowriders tratan de redimir el estigma vinculado al pachuco y limpiar la imagen que se les ha imputado por añadidura. Los lowriders generan unas narrativas de redención del pachuco en las que es posible identificar una relación más cercana con esta simbología, de modo que los presentan como personas honorables y dignas de respeto: "el pachuco era un dandy, era una vestimenta de un personaje bien vestido, muy elegante" (Jinete, entrevista) (Del Monte, 2012). Finalmente, esta redención ocurre en perjuicio del otro estigma
}

prácticas - entenderlas como formas de automovilidad que se llevan a cabo a través de los carros antiguos modificados en la ciudad de Tijuana- y los significados que adquieren para la articulación de las adscripciones lowrider. Los resultados que se presentarán, en última instancia, se refieren a una expresión nostálgica y memoriosa de carros antiguos que han sido modificados y arreglados para circular por la ciudad y que funge como un elemento de inscripción social. El tipo de movilidad que por lo general se concibe desde un automóvil es un movimiento horizontal, es decir, se presupone que el carro genera movimientos para adelante y/o para atrás y hacia la derecha y/o a la izquierda. Sin embargo, existen otros tipos de movimiento que pueden realizarse desde los automóviles, como los característicos de los lowriders. En el trabajo etnográfico se han explorado tres automovilidades lowrider en la ciudad de Tijuana: la horizontal desacelerada o lenta, la vertical y la temporal, las cuales se asocian, respectivamente, a tres prácticas que en su conjunto articulan el reconocimiento de las identidades lowrider: exhibición, contemplación y evocación.

\section{AUTOMOVILIDAD HORIZONTAL DESACELERADA. LENTOS}

La primer automovilidad de los lowriders alude a la práctica de manejar de manera calmosa y pausada. Mientras el tránsito vehicular fluye de manera constante y apresurada, los lowriders manejan con parsimonia por las calles de Tijuana, lo que representa una transgresión para el sistema urbano que está abocado a dirigir la circulación de flujos y movimientos de forma rápida y ordenada. La

\footnotetext{
que acarrean, el del cholo, del cual aceptan su influencia simbólica, pero se deslindan de sus prácticas, que continúan tratando de manera estereotipada: "el cholo ya pasó [...] fue una moda, fue una época y ya precisamente, todo ese cholo, pues tenía que crecer, tenía que madurar" (Rancho, entrevista) (Del Monte, 2012).
} 
movilidad desacelerada en los lowriders irrumpe en el espacio público de modo deliberado y enfatiza la exhibición de sus prácticas. Manejar lento para los lowriders implica llamar la atención de los demás sujetos que circulan en la ciudad y que - absortos en su monotonía cotidiana- pasan por alto muchas situaciones de su entorno. Así, circular lentamente implica hacerse reconocer como lowriders en el espacio urbano de Tijuana. Al manejar suavecito y "tumbado" - rasgo fundamental de la actitud del cholo-, el lowrider exhibe sus características de manera performática y solemne. La movilidad desacelerada funciona como estrategia para comunicar la identidad lowrider y sus prácticas, como sucede cuando anuncian el "baile anual". Se trata de las reminiscencias de una práctica que se remonta a las formas propagandísticas de una época en que las noticias se difuminaban de voz en voz por las calles del barrio y consiste en la circulación de paso lento y fastuoso acompañado de música y de una grabación que anuncia el acontecimiento:

Damos una vuelta, paramos, llevamos música, llevamos un pregrabado del evento y los carros van todos en caravana pasándola bien [...] Anunciamos el baile, paramos en las esquinas y bajamos los cartelones y ofrecemos boletos. Damos toda la vuelta, siempre le metemos unas cuatro o cinco horas, es como por la colonia (Tacha, entrevista) (Del Monte, 2012). ${ }^{6}$

Con la movilidad desacelerada, los lowriders se afirman en el espacio público a partir de una práctica performática que enfatiza la exhibición de su identidad.

\footnotetext{
${ }^{6}$ Respecto de la muestra de entrevistados, para cumplir con el criterio de heterogeneidad se tomó en cuenta el tiempo en el club de lowriders y la posición en el mismo. De manera que la mitad de los entrevistados en el trabajo de campo tenían más de 15 años en el club y más de 40 años de edad, en tanto que la permanencia en el club de la otra mitad era menor y rondaba entre los 25 y 35 años de edad. La intención fue contar con una muestra de informantes que pudiera ofrecer un panorama amplio y completo sobre las formas en que viven y significan el hecho de ser bikers y lowriders. Todos los entrevistados fueron hombres.
}

\section{AUTOMOVILIDAD VERTICAL. ESTÉTICOS}

Con el término "automovilidad vertical" se hace referencia a un tipo de movimiento que los lowriders realizan, como su nombre lo insinúa, desplazando el carro de arriba para abajo. Esta movilidad se logra con la colocación de un complejo sistema de levantamiento hidráulico instalado en los amortiguadores de las llantas y de una serie de baterías en la cajuela que lo apoyan con la energía y que les permite levantar la carrocería del automóvil antiguo varios centímetros por encima del piso. La colocación de hidráulicos es un elemento distintivo del fenómeno lowrider. Algunos han pensado esta situación como una forma de "vida" que adquiere el carro, ya que de esta manera puede "bailar" (Bright, 1994). El carro que está "vivo" y se mueve de arriba para abajo representa otra estrategia de manifestar la identidad lowrider, pero con un énfasis distinto al anterior.

Si la colocación de sistemas hidráulicos comenzó como una forma de resistencia ante la vigilancia de la autoridad en Estados Unidos, es necesario percibir de qué manera se ha resignificado dicha práctica de modificación de los carros en Tijuana. En el trabajo de campo se encontró que el movimiento que posibilitan los hidráulicos no tiene fines de desplazamiento territorial, sino de contemplación visual. Si la movilidad desacelerada hace hincapié en la exhibición, la movilidad vertical enfatiza la contemplación. Mientras la primera representa un grito en el espacio público para llamar la atención, la segunda busca mantenerla. Finalmente, ambas son estrategias que producen un reconocimiento identitario. De ahí que la movilidad vertical que estamos analizando se manifieste como una movilidad estética. Emplear sistemas hidráulicos no tiene fines utilitarios, sino estéticos: "Cuando bajas este carro por atrás, no sé si te has fijado que lo subo, parece un gatito con sus patas para enfrente y se miran bonito, siempre. Este carro es clásico y siempre tienes que traer hidráulicos o airbags para que se miren mejor" (House, entrevista) (Del Monte, 2012). 


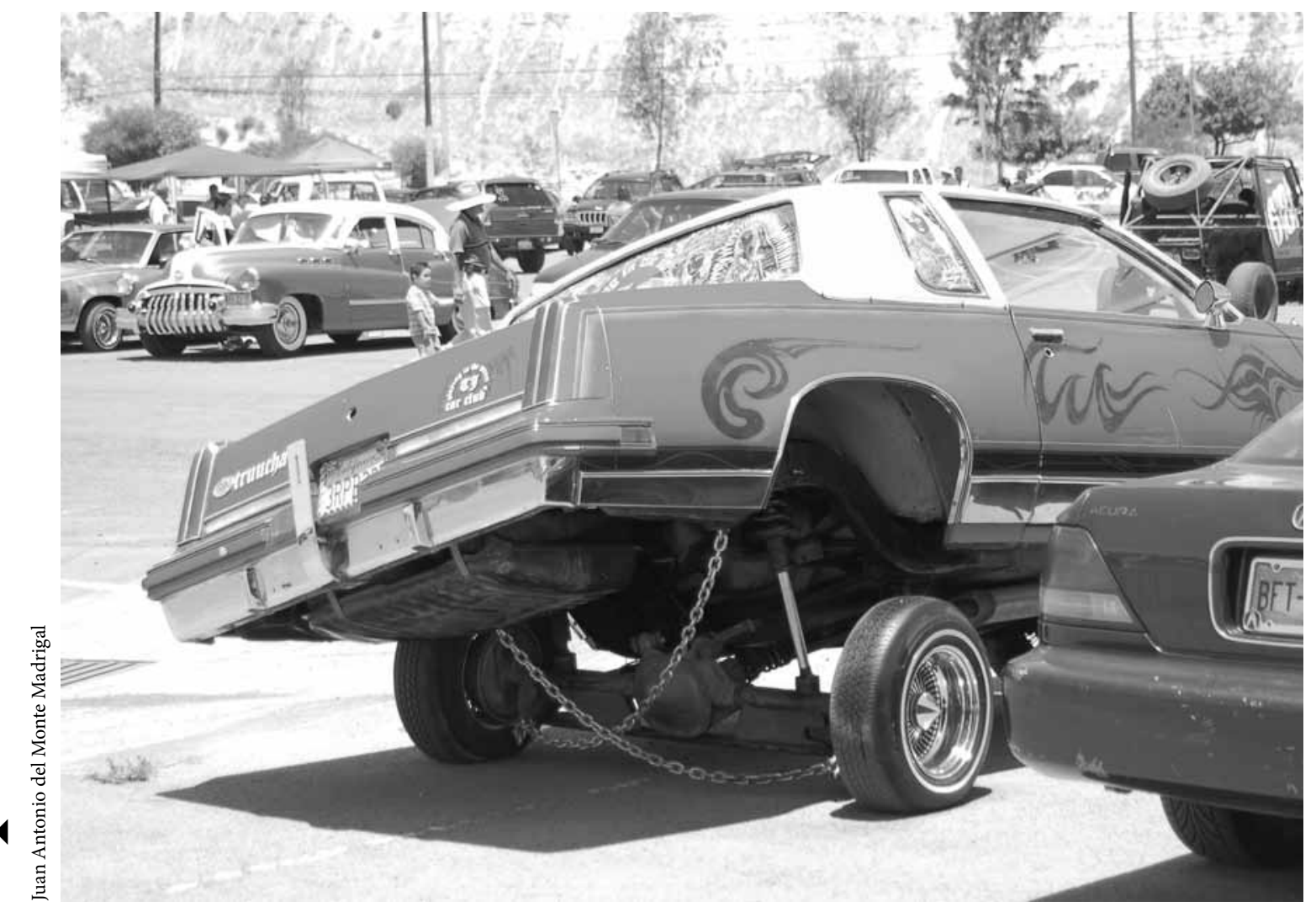

Lowriders, 2012.

La colocación de sistemas hidráulicos es también una muestra de movimiento, aunque es un movimiento que no está contemplado originalmente en la fabricación del carro. El automóvil circula hacia enfrente, hacia atrás o hacia los lados, pero el lowrider también lo desplaza de arriba a abajo, el lowrider hace brincar su carro. En este sentido representa una tergiversación y un cambio en las formas en que entendemos la movilidad en carro. El carro lowrider es entonces una transgresión estética al flujo de la automovilidad. Ondine Chavoya (2004) hace un análisis de la instalación artística La ranfla cósmica, de Rubén Ortiz-Torres, y su relación con la cultura del lowrider en el sur de California. Alude al carácter estético de las prácticas del lowrider, menciona que desde sus inicios el lowrider fue visto como un movimiento tanto étnico como estilístico en el que las características estéticas del carro funcionaban como una táctica de resistencia étnica. Y cita a Bright cuando dice: "la estética del carro le dio voz a sus diferencias" (Bright et al., 2000: 41). El mismo Ortiz-Torres ha declarado que los lowriders "son la exposición estética más lograda de la cultura del carro" (OrtizTorres, 2000: 37).

Ahora bien, esta vertiente estética es la que predomina en la expresión lowrider en la ciudad de Tijuana, como podemos ver a través del análisis de su movilidad vertical. El fenómeno lowrider de modificación de carros clásicos en Tijuana está actualmente más ligado a la estética — de la nostalgia, en este caso- que a la reivindicación étnica, cuyo sentido se ha diluido en otras expresiones reivindicatorias. De este modo, si existen otras formas 
de reivindicación más claras de este fenómeno en Tijuana, su objeto está relacionado en todo caso con una reivindicación de las culturas populares de barrio. Situación que, por supuesto, no está exenta de implicaciones étnicas. Cuando los lowriders manejan lentos y verticales ejercen una práctica de resistencia social que visibiliza en el espacio público su historia y sus características identitarias como fenómenos populares y estigmatizados. Al performar sus identidades de esta manera reivindican y reposicionan una memoria social, vinculada a las culturas populares, que ha sido relegada y estereotipada en la narrativa predominante sobre la población tijuanense. ${ }^{7}$

\section{AUTOMOVILIDAD TEMPORAL. MEMORIOSOS}

Los lowriders que circulan en la ciudad toman como plataforma el carácter geográfico de la automovilidad - la circulación en las calles- para proyectar una movilidad que no es espacial sino temporal. La automovilidad temporal lowrider es evocativa del tiempo mismo. Cuando los lowriders exhiben sus carros clásicos en las calles de Tijuana están evocando el "lugar de la memoria" al que está asociada su identidad: "La memoria es un fenómeno perpetuamente actual, un vínculo que nos ata al eterno presente; la historia es una representación del pasado" (Nora, 1989: 8). Es decir, mientras que la historia es una reconstrucción de lo que ya no es, y por tanto está representada como un pasado lejano, la memoria es un elemento en el presente que está vivo y es dinámico, es aquello del pasado que sigue actuando en el presente. Los "lugares de la memoria" no se reducen a ser sitios topográficos donde se

\footnotetext{
${ }^{7}$ La memoria a la que se alude se relaciona en mayor medida con las formas expresivas vinculadas a grupos juveniles de clase baja en la frontera, como pachucos y cholos, y que han sido excluidos de un discurso oficialista enfocado en visibilizar las agrupaciones juveniles de la clase alta (Villacorta, 1989).
}

ha anclado la memoria, sino que se refieren a una configuración cultural en la que personas, acontecimientos, objetos y prácticas son referentes de un pasado que sigue ordenando el presente, marcas externas en que se apoyan las prácticas sociales (Ricoeur, 2006). La automovilidad temporal de los lowriders está ligada a la nostalgia. La nostalgia es el motivo que guía los sentidos del actuar del lowrider que se analizó, el cual encuentra su objetivación en la movilidad memoriosa. Cuando sacan sus automóviles antiguos a las calles "evocan" un pasado al que honran y hacen justicia al traerlo al presente. Así, la movilidad temporal "activa" la memoria del pasado y la inscribe en el presente. Cuando circula una lenta y extensa fila de 15 o 20 ranflas $^{8}$ la gente voltea porque los autos resaltan del fluir "normal" de las calles de Tijuana, pues no todo el tiempo se ven carros clásicos modificados al estilo lowrider que desfilan con parsimonia y en caravana por las avenidas de la ciudad:

es algo bien grandioso, el subirte a tu clásico y manejarlo y ver cómo jalas miradas más que como cuando vas en un carro nuevo, la raza hasta voltea, ha habido personas que me salen en el camino corriendo, "pérese, oiga, pérese, oiga" [...] la señora quería tomarse unas fotos porque su marido había tenido uno igual que el mío (Cerillo, entrevista) (Del Monte, 2012).

Los carros lowrider - al performar sus prácticas de conducir - remiten a otra época y así, conduciendo, nos comunican sus "lugares de la memoria":

es una comunicación, fíjate, sin querer en esa forma aportamos información a gente [...]. Hasta recuerdan los carros: "Uy, yo tenía uno como ése, me llevé a mi esposa en él". Platican señores ya más con otros muchos años más que yo, no sé, 20 años más que yo por decir así: "Uy, si yo te contara”, entonces es suave, es una interacción muy buena, muy amena (Tacha, entrevista) (Del Monte, 2012).

8 "Ranfla" es el término que utilizan los lowriders para referirse a los automóviles antiguos modificados. También les llaman "carruchas". 
Por último, es preciso recalcar que el significado asignado a esta automovilidad es central en la constitución de sus identidades: "es algo que cuando tengas un clásico lo vas a entender [...] así estamos nosotros de los clásicos, enamorados de nuestras ranflas" (Cerillo, entrevista) (Del Monte, 2012). De este modo, si la movilidad desacelerada enfatiza la exhibición y la movilidad vertical se enfoca en la contemplación estética, la movilidad temporal hace lo propio en lo que corresponde a la evocación. Simbólicamente, el lowrider con sus carros clásicos nos hace "viajar en el tiempo" cuando circula por la ciudad y nos impulsa a evocar un "lugar de la memoria" de la historia de Tijuana. Esta atmósfera de tiempo suspendido quizá sólo sea más perceptible en Cuba, que a partir del triunfo de la revolución y el consecuente embargo comercial se ha mantenido con puros automóviles de mediados de siglo (Narotzky, 2002). Ahora bien, cuando se evoca la memoria de este pasado con prácticas de circulación como la automovilidad temporal se pone en operación un mecanismo narrativo que relata activamente una historia a veces olvidada frente a las versiones dominantes, el devenir de fenómenos populares y subalternos en la frontera, como el caso de las identidades en cuestión. Así, la memoria que activan los lowriders al circular, más allá de ser la reproducción pasiva y acrítica de un pasado, representa la búsqueda activa de su posicionamiento social.

\section{ESTÉTICAS DE LA NOSTALGIA}

El análisis de las automovilidades lowrider revela que el sentido que predomina en las prácticas de modificación de autos antiguos es principalmente estético. De hecho, ese sentido estético se encuentra anclado históricamente al movimiento chicano y la recuperación que hizo del muralismo mexicano y su simbología como medio de comunicación artística, social y política (Valenzuela, 1998; Bright, 1998; Bright et al., 2000). Los lowriders han hecho una gran aportación al arte urbano al ponerle cuatro ruedas a los murales, que muchas veces son pintados en las puertas y los cofres de sus carros. ${ }^{9}$ Pero la movilidad estética que sin duda los distingue es el movimiento ascendente y descendente que logran a partir del sistema hidráulico. Así, la expresión estética del lowrider es la que predomina actualmente en las calles de Tijuana, como una forma de afirmar públicamente su identidad. De este modo, la larga tradición del movimiento de resistencia chicano se manifiesta también en el performance de los lowriders. Las automovilidades lowrider forman parte del esfuerzo por buscar el reconocimiento de una simbología estigmatizada, como la relacionada con los pachucos y los cholos. Los lowriders, ubicados en la historia de prejuicios y descréditos hacia la iconografía relacionada con el pueblo chicano, han logrado un reconocimiento de su identidad a partir de sus movilidades en la ciudad. Pero hay que hacer una advertencia - que debe ser desarrollada en posteriores trabajos-, la descalificación a la que se han visto sometidos históricamente no es unilateral, sino que ha venido de ambos países. Así, frente a las desaprobaciones del estilo chicano por parte de los valores hegemónicos estadounidenses $\mathrm{y}$ frente a las narrativas alienizadoras que desde el centro de México se tenían sobre la frontera, los lowriders en Tijuana han enarbolado la nostalgia y la simbología popular-mexicana como una forma de reconstrucción identitaria y de rearticulación histórica de las culturas populares fronterizas.

De esta manera, el análisis de las automovilidades de los lowriders muestra una estética de la nostalgia que podemos entender como un mecanismo performático de la memoria lowrider que establece una relación con el pasado desde la sociedad actual. Los lowriders se exhiben para ser contemplados y así evocar el "lugar de la memoria" que los conforma. Exhibición, contemplación y evocación son los elementos de la estética de la nostalgia que organizan

\footnotetext{
${ }^{9}$ De hecho, los artistas tijuanenses dedicados a la modificación, carrocería y pintura de carros han tenido una gran influencia en los lowriders del otro lado de la línea fronteriza (Tatum, 2011).
} 


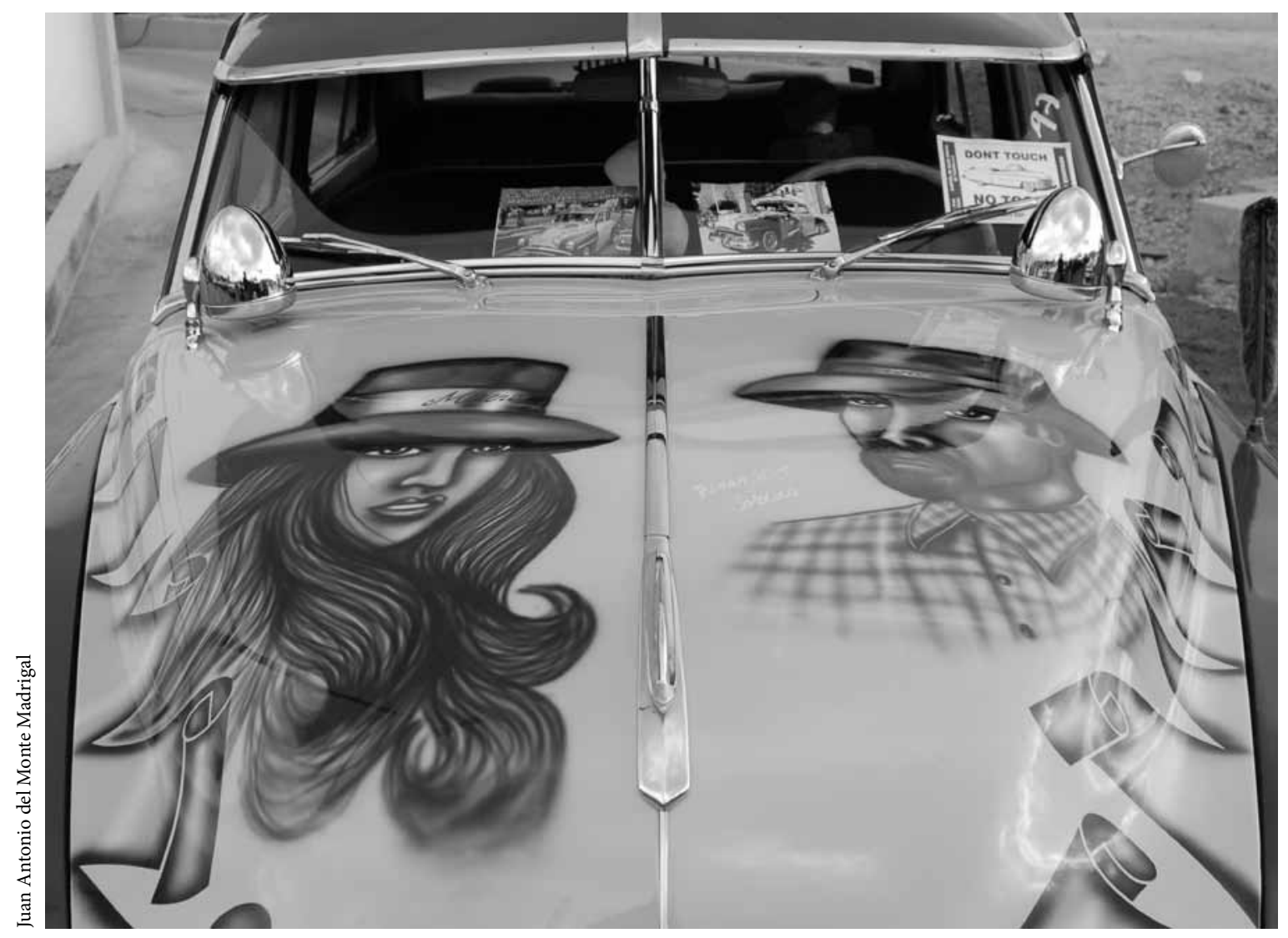

Lowriders, 2012.

la manifestación y el reconocimiento identitario de los lowriders en Tijuana. Estas tres automovilidades - lenta, vertical y temporal- integran el performance de la identidad lowrider en esta ciudad. Michel de Certeau entendía la memoria como una "ocasión" para actuar, para poner en "práctica" el pasado histórico que está latente en el recuerdo. La memoria, escribe, "muy lejos de ser el relicario o el bote de basura del pasado, vive de creer en lo posible y en esperarlo, vigilante, al acecho" (De Certeau, 1996: 96). Con ello puso en evidencia el potencial del pasado, no sólo para reproducirlo, sino, como dice François Dosse, "como fuente de inspiración para verdaderas creaciones en las fases de crisis y conmoción de lo instituido, como posible recurso para otra gramática de nuestra relación con el mundo" (Dosse, 2009: 90). Performar las automovilidades a través de una estética de la nostalgia se constituye como una práctica reivindicatoria de la identidad lowrider en el presente y el futuro, que es pura posibilidad. Así, lo que se busca con las automovilidades lowrider, sus significados y su exhibición pública por las calles de la ciudad es la afirmación de una identidad sobajada históricamente, es la apropiación de espacios sociales por medio de actos performáticos que transgreden la "normalidad" de la vida cotidiana.

Habría que concluir, a manera de provocación, con un último señalamiento. El énfasis en la exhibición, contemplación y evocación de los significados de la identidad lowrider que irrumpen en la vida cotidiana de la ciudad es también la posibilidad de 
apropiarse de las calles y la consecuente disputa por el espacio urbano. La construcción social de los significados del espacio citadino mediante la interacción automovilizada es asimismo una declaración de los principios de uso de las calles, es una postura frente a otras movilidades urbanas que también disputan los sentidos de utilizar las calles - como peatones y ciclistas-. Con ello habría que advertir que frente a las concepciones que perciben los espacios de tránsito - calles y avenidas- como "no lugares" o "espacios del anonimato", la búsqueda de reconocimiento al performar la identidad lowrider implica llenar de significados los espacios urbanos, incluidos aquellos destinados a la circulación y la interacción efímera.

\section{BIBLIOGRAFÍA}

Alexander, Jeffrey, 2011, Performance and Power, Polity, Cambridge.

Amin, Ash y Nigel Thrift, 2002, Cities. Reimagining the Urban, Polity, Cambridge.

Bauman, Zygmunt, 2001, "Identity in the Globalising World", en Social Anthropology, European Association of Social Anthropologists, vol. 9, núm. 2, pp. 121-129.

Bright, Brenda, 1994, "Mexican American Low Riders: An Anthropological Approach to Popular Culture", tesis de doctorado, Rice University, Houston.

_ 1998, "Nightmares in the New Metropolis. The Cinematic Poetics of Low Riders", en Joe Austin y Michael Nevin, Generations of Youth. Youth Cultures and History in Twentieth-Century America, New York University Press, Nueva York.

- et al., 2000, Customized: Art Inspired by Hot Rods, Low Riders and American Car Culture, Institute of Contemporary Art, Boston.

Certeau, Michel de, 1996, La invención de lo cotidiano I. Artes de hacer, Universidad Iberoamericana, Centro Francés de Estudios Mexicanos y Centroamericanos, Instituto Tecnológico y de Estudios Superiores de Occidente, México.

Chavoya, Ondine, 2004, "Customized Hybrids: The Art of Rubén Ortiz-Torres and Lowriding in Southern California”, en The New Centennial Review, Michigan State University Press, vol. 4, núm. 2, pp. 141-184.
Cresswell, Tim, 2006, On the Move. Mobility in the Modern World, Routledge, Nueva York.

Dosse, François, 2009, Paul Ricoeur y Michel de Certeau. La historia: entre el decir y el hacer, Nueva Visión, Buenos Aires.

Featherstone, Mike, Nigel Thrift y John Urry (eds.), 2005, Automobilities, SAGE, Londres, Thousand Oaks, Nueva Delhi.

Ganahl, Pat, 2000, “The Hot Rod Culture”, en Brenda Bright et al., Customized: Art Inspired by Hot Rods, Low Riders and American Car Culture, Institute of Contemporary Art, Boston.

García Canclini, Néstor, Alejandro Castellanos y Ana Rosas Mantecón, 1996, La ciudad de los viajeros. Travesías e imaginarios urbanos: México, 1940-2000, Grijalbo, México.

Gradante, William, 1982, "Low and Slow, Mean and Clean”, en Natural History, vol. 91, núm. 4, pp. 28-39.

Giménez, Gilberto, 2004, “Culturas e identidades", en Revista Mexicana de Sociología, vol. 66, número especial, pp. 77-99.

- 2009, Identidades sociales, Consejo Nacional para la Cultura y las Artes, Instituto Mexiquense de Cultura, México.

Instituto Nacional de Estadística y Geografía (INEGI), Censo de Población y Vivienda 2010, en línea: <www. inegi.org.mx>.

Miller, Daniel, 2001, Car Cultures. Materializing Culture, Berg, Oxford.

Monte Madrigal, Juan Antonio del, 2012, "Identidades motorizadas. Las culturas urbanas de bikers y lowriders en la frontera de Tijuana", ponencia, $5^{\circ}$ Congreso Internacional de Sociología "Espacios Contestatarios", 25-28 de septiembre, Ensenada [entrevistas a Cerillo, House, Jinete, Rancho y Tacha].

Narotzky, Viviana, 2002, "Our Cars in Havana”, en Peter Wollen y Joe Kerr (eds.), Autopia. Cars and Culture, Reaktion Books, Hong Kong.

Nora, Pierre, 1989, "Between Memory and History: Les Lieux de Mémoire”, en Representations, núm. 26, pp. 7-25.

Ortiz-Torres, Rubén, 2000, "Cathedrals on Wheels", en Brenda Bright et al., Customized: Art Inspired by Hot Rods, Low Riders and American Car Culture, Institute of Contemporary Art, Boston.

Padilla, Antonio, 1998, Inicios urbanos del norte de Baja California. Influencias e ideas. 1821-1906, Instituto de Investigaciones Históricas-Universidad Autónoma de Baja California, Mexicali.

Plascencia, Luis, 1983, "Low Riding in the Southwest: Cultural Symbols in the Mexican Community”, en Mario 
Garcia et al. (eds.), History, Culture and Society: Chicano Studies in the 1980s, National Association for Chicano Studies, Bilingual Press-Editorial Bilingüe, Ypsilanti.

Ramos, Jorge, 2010, "Cambiamos el paradigma de gobierno..., en Manuel Acuña y Mario Ortiz, Nuestro destino común. El Programa Integral de Repavimentación de Tijuana (Pire). Memoria documental de una experiencia de gobierno, Ayuntamiento de Tijuana, Tijuana.

Ricoeur, Paul, 2006, Memory, History, Forgetting, The University of Chicago Press, Chicago.

Sandoval, Denise, 2003, "Bajito y suavecito: The Lowriding Tradition”, en línea: <http://latino.si.edu/virtualgallery/lowrider/lr_sandovalessay.htm $>$.

Sheller, Mimi y John Urry, 2000, "The City and the Car", en International Journal of Urban and Regional Research, vol. 24, núm. 4, pp. 737-757.

Tatum, Charles, 2011, Lowriders in Chicano Culture: From Low to Slow to Show, ABC-CLIO, Santa Barbara.

Taylor, Charles, 1996, "Identidad y reconocimiento", en Revista Internacional de Filosofía Política, núm. 7, pp. 10-19.

Thrift, Nigel, 2004, "Movement-Space: The Changing Domain of Thinking Resulting from the Development of New Kinds of Spatial Awareness", en Economy and Society, Routledge, vol. 33, núm. 4, pp. 582-604.
2005, "Driving in the City", en Mike Featherstone, Nigel Thrift y John Urry (eds.), Automobilities, SAGE, Londres, Thousand Oaks y Nueva Delhi.

Urry, John, 2007, Mobilities, Polity, Cambridge.

Valenzuela Arce, José Manuel, 1988, ;A la brava ese! Cholos, punks, chavos banda, El Colegio de la Frontera Norte, Tijuana.

, 1998, El color de las sombras. Chicanos, identidad y racismo, El Colegio de la Frontera Norte, Universidad Iberoamericana, Plaza y Valdés, México.

(coord.), 2000, Decadencia y auge de las identidades. Cultura nacional, identidad cultural y modernización, El Colegio de la Frontera Norte, Plaza y Valdés, México.

, Alfredo Nateras y Rossana Reguillo (coords.), 2007, Los maras. Identidades juveniles al límite, Universidad Autónoma Metropolitana, Juan Pablos, El Colegio de la Frontera Norte, México.

Villacorta, Mario, 1989, "Grupos sociales y de servicio", en Jesús Ortiz y David Piñera, Historia de Tijuana 1889-1989. Edición conmemorativa del centenario de su fundación, Universidad Autónoma de Baja California, Gobierno del Estado de Baja California, XII Ayuntamiento de Tijuana, Tijuana.

Wollen, Peter y Joe Kerr (eds.), 2002, Autopia. Cars and Culture, Reaktion Books, Hong Kong. 